\title{
ORIGINAL RESEARCH \\ The Woven EndoBridge: A New Aneurysm Occlusion Device
}

\begin{abstract}
Y.H. Ding
D.A. Lewis

R. Kadirvel

BACKGROUND AND PURPOSE: The WEB device is an intrasaccular ellipsoid braided-wire embolization device designed to provide flow disruption along the aneurysm neck. The purpose of this study was to evaluate, in an in vivo aneurysm model, the acute and chronic performance of the WEB device regarding immediacy, degree, and durability of aneurysm occlusion.
\end{abstract}

D. Dai

D.F. Kallmes

MATERIALS AND METHODS: The WEB device was implanted in 24 elastase-induced aneurysms in New Zealand white rabbits and followed for $1,3,6$, and 12 months ( $n=6$ at all time points). Degree of intra-aneurysmal flow disruption was graded on a 4-point scale based on DSA within 10 minutes following device implantation. Chronic aneurysm occlusion was rated by using a 3-point scale. All aneurysms were harvested for histologic analysis.

RESULTS: Immediate postimplant grade 1 (complete flow cessation) was noted in 7 (29\%) of 24 cases. Grade 2 (near-complete flow cessation) was noted in $13(54 \%)$ of 24 cases. At follow-up, complete occlusion was noted in 8 (33\%) of 24 cases. Near-complete aneurysm occlusion was noted in 14 (58\%) of 24 cases, while incomplete occlusion was noted in $2(8 \%)$ cases. Stable aneurysm occlusion was present in 7 (29\%) of 24 cases; progressive occlusion, in 14 (58\%); and recanalization, in $3(13 \%)$ cases. Histologic findings included aneurysm cavities filled with organized thrombus with connective tissue across the aneurysm neck.

CONCLUSIONS: The WEB device in experimental aneurysms demonstrated promising rates of immediate and long-term aneurysm occlusion.

ABBREVIATIONS: DSA $=$ digital subtraction angiography; $\mathrm{HE}=$ hematoxylin and eosin; $\mathrm{WEB}=$ Woven EndoBridege
$\mathbf{R}$ ecent advances in endovascular aneurysm repair have substantially impacted our understanding of how treated aneurysms heal after embolization. Until recently, the primary focus of most aneurysm devices was on healing within the aneurysm sac. Bare platinum coils, subject to high rates of aneurysm recanalization, were modified to enhance intrasaccular organization. Unfortunately, it has yet to be proved in the clinical setting that most coil modifications perform better than bare platinum coils. ${ }^{1-10}$

Intraluminal "flow diverters," comprising tubular braided metallic implants, have shown, in preclinical studies and in early clinical reports, the ability to achieve complete occlusion in a high proportion of aneurysms. ${ }^{11-20}$ Excellent occlusion rates after flow-diverter therapy are seen even in large and giant aneurysms, which show recanalization rates of $\geq 50 \%$ after coil embolization. ${ }^{21,22}$ The success of these new devices has refocused the attention from the aneurysm dome to the aneurysm neck as the point of relevance in aneurysm treatment. Specifically, achieving a stable construct across the aneurysm neck, irrespective of intra-aneurysmal findings, may be the best predictor of long-term occlusion.

Notwithstanding the early promise of intraluminal flow diverters, important shortcomings limit widespread application of these devices. Because of the perceived risk of thromboembolism, patients treated with endoluminal flow diverters

Received June 4, 2010; accepted after revision September 25

From the Neuroradiology Research Laboratory, Department of Radiology, Mayo Clinic, Rochester, Minnesota.

This study was funded by Sequent Medical, Aliso Viejo, California.

Please address correspondence to David F. Kallmes, MD, Department of Radiology, Mayo Clinic, 200 First St SW, Rochester, MN, 55905; e-mail: kallmes.david@mayo.edu DOI 10.3174/ajnr.A2399 are placed on dual antiplatelet therapy. This medical therapy limits use, in most cases, to unruptured aneurysms, given the risk of hemorrhagic complications if patients require external ventricular drainage. ${ }^{23}$ Use of these endoluminal devices is further limited in ruptured aneurysms because closure of the aneurysm is delayed by weeks or months after implantation, such that an acutely ruptured aneurysm would not be adequately protected in the short term. Furthermore, the design of the endoluminal devices renders them difficult to use in bifurcation-type aneurysms. Last, use of these devices in the vicinity of eloquent perforating arteries raises concern for stroke.

In this report, we detail a new device that mimics the flowdiverting mechanism of the intraluminal flow diverters, yet is placed entirely within the aneurysm cavity. This new intrasaccular device, termed the WEB aneurysm embolization device (Sequent Medical, Aliso Viejo, California), achieves placement of a high-attenuation metallic mesh both along the aneurysm wall and across the neck, which thus functions as an intrasaccular flow diverter. The device is intended as a standalone therapy, without adjunctive coils placed into the aneurysm cavity or within the WEB device. Because the WEB device is intrasaccular and presents a metal mesh-filled neck not unlike a coil-packed aneurysm, we anticipate that there will be no need for antiplatelet therapy. Further, the device likely would be amenable for treatment of a broad range of aneurysms, including bifurcation-type morphologies, and does not place adjacent perforating arteries at risk. Last, the WEB device is designed to facilitate acute aneurysm occlusion, rendering it appropriate for use in ruptured aneurysms.

The purpose of this study was to evaluate, in an in vivo aneurysm model, the acute and long-term performance of the 


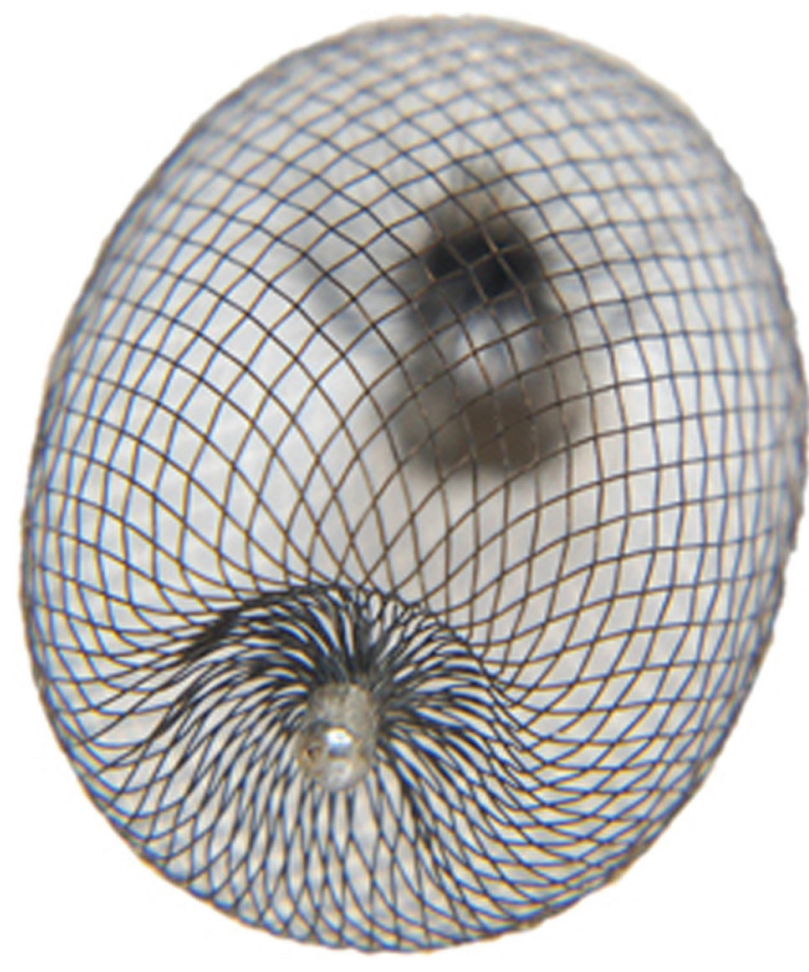

Fig 1. Photograph of the WEB device. The proximal and distal ends of the device have radiopaque markers attached.

WEB device regarding immediacy, degree, and durability of aneurysm occlusion.

\section{Materials and Methods}

The WEB aneurysm embolization device is constructed of braided nitinol wire with proximal and distal platinum markers that hold the structure in a globular shell shape as shown in Fig 1. The wire mesh presents a consistent pore structure for spanning an aneurysm ostium with between $35 \%$ and $45 \%$ metal coverage. The devices used in this study were designed to have a maximum interstrut distance of $200 \times$ $540 \mu \mathrm{m}$. The device is delivered in fashion similar to that in the MicroCoil system (Micrus, San Jose, California), attached to the distal end of a detachment system, through a microcatheter. The detachment system is electrothermal, providing instantaneous detachment. The mesh structure of the WEB provides a stentlike adherence to the aneurysm wall. This adherence, in contrast to smooth nonadherent balloons previously used for aneurysm occlusion, may provide device stability within the aneurysm. ${ }^{24}$

\section{In Vivo Experiments}

Aneurysms $(n=24)$ were created as previously described. ${ }^{25}$ No preor periprocedural antiplatelet therapy was used. At least 3 weeks passed between aneurysm creation and embolization. ${ }^{26}$ Anesthesia was induced by using $74 \mathrm{mg} / \mathrm{kg}$ of ketamine, $5 \mathrm{mg} / \mathrm{kg}$ of xylazine, and $1 \mathrm{mg} / \mathrm{kg}$ of acepromazine and was maintained by using ketamine (10 $\mathrm{mg} / \mathrm{kg}$ ) and xylazine ( $3 \mathrm{mg} / \mathrm{kg}$ ). A surgical cut-down of the right femoral artery was performed, and a $5 \mathrm{~F}$ sheath was inserted. A $5 \mathrm{~F}$ guide catheter (Envoy; Cordis, Miami Lakes, Florida) was placed into the aortic arch, and DSA was performed. Heparin (500 U intravenously) was administered, and then a microcatheter (Renegade Hi-Flo; Boston Scientific, Natick, Massachusetts) was placed over a microguidewire (Transend EX; Boston Scientific) into the aneurysm cavity.
Device Sizing. A variety of diameters and lengths of the WEB device are available so that the physician can select the device that will best fit the aneurysm. The radial strength of the device is comparable with a coil mass, and it is of essentially constant volume over a range of expanded diameters (unpublished data, Sequent Medical, 2009) such that if the device is oversized relative to the aneurysm, it can be elongated to avoid overexpansion and rupture of the aneurysm.

The WEB size was selected to match the aneurysm size with a diameter that at least matched the greatest aneurysm width and had nearly the same height. The wire was removed, and the WEB device was advanced into the aneurysm cavity and deployed. The microcatheter was removed, and DSA of the aortic arch and brachiocephalic vessels was performed up to 10 minutes following device implantation. Subjects were followed for 1 month $(n=6), 3$ months $(n=6)$, 6 months $(n=6)$, and 1 year $(n=6)$. At the time of death, animals were deeply anesthetized. DSA of the aortic arch and the abdominal aorta was performed. Animals were then humanely killed with a lethal injection of pentobarbital. Harvested aneurysms and aortas were immediately fixed in $10 \%$ neutral buffered formalin.

\section{Data Analysis}

Angiographic Evaluation. Aneurysm dimensions (neck width, aneurysmal height and width) were determined with DSA measurements, which were adjusted by using an external sizing device of known diameter. Angiographic evaluation was performed by angiogram analysis conducted immediately after device implantation as well as prior to death.

Immediate Angiographic Analysis. The purpose of detailed immediate angiographic analysis was to determine the likelihood that the WEB device would achieve enough flow arrest within the aneurysm to allow clinical application in ruptured aneurysms. We envision that clinical use in ruptured aneurysms will be accepted only if the device can achieve complete or near-complete flow arrest in most cases, to protect the patient from early aneurysm rerupture. Given the novel nature of the WEB device and its dissimilarity to coils, we are required to propose an untested aneurysm occlusion score based on intra-aneurysmal flow rather than the degree of coil occlusion. Using the immediate postimplantation DSA images, obtained 5-10 minutes after implantation, we assessed the degree of intra-aneurysmal flow disruption by using a 4-point scale, including grade 1 (complete cessation of flow), grade 2 (near-complete cessation of flow), grade 3 (persistent flow into the aneurysm with moderate-to-marked stasis), and grade 4 (persistent flow with no or minimal disruption within the aneurysm). At follow-up, we graded the degree of aneurysm occlusion by using a 3-point scale: grade I, complete occlusion; grade II, near-complete occlusion; and grade III, incomplete occlusion. Interval change was assessed by comparing the 3-point filling scale results of individual aneurysms immediately after implantation by using a separate 3-point scale indicating that the aneurysm was stable, recanalized, or progressively thrombosed.

\section{Conventional Histopathologic Processing}

After routine tissue processing, the fixed samples were embedded in paraffin. Samples were then cut at $1000-\mu \mathrm{m}$ intervals in a coronal orientation, permitting long-axis sectioning of the aneurysm neck, with use of an IsoMet Low-Speed Saw (Buehler, Lake Bluff, Illinois). The device segments were carefully removed under a dissecting microscope. The samples were then re-embedded in paraffin, sectioned at 5-6 $\mu \mathrm{m}$, and stained with HE. 

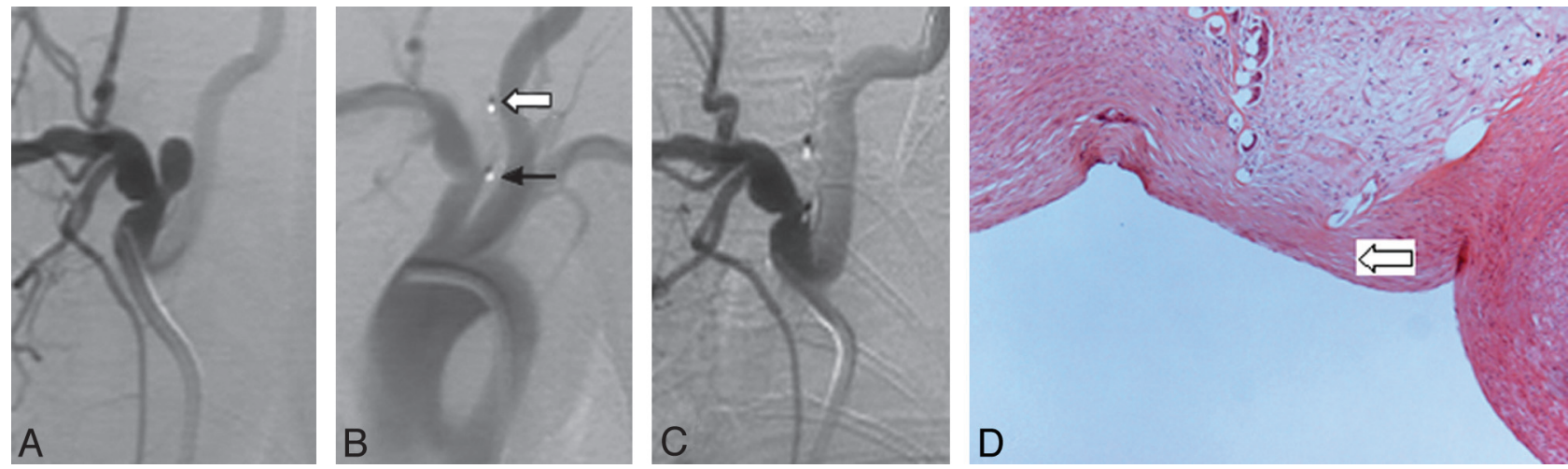

Fig 2. A, Anteroposterior DSA before treatment. B, DSA after device deployment shows complete immediate cessation of flow. Note radiopaque distal (block arrow) and proximal markers (black arrow) denoting the ends of the device. C, DSA at 3 months shows complete aneurysm occlusion. $D$, Photomicrograph (HE, original magnification $\times 100)$ shows organized connective tissue within the aneurysm lumen and attenuated connective tissue across the aneurysm neck (block arrow).
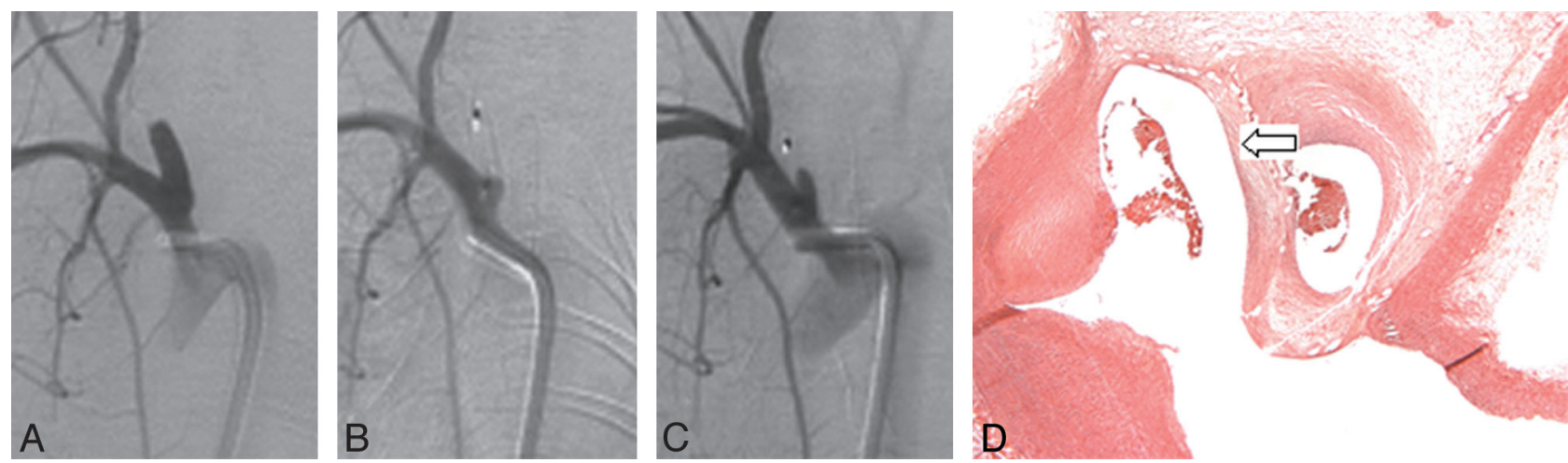

Fig 3. $A$, Anteroposterior DSA before treatment. $B$, DSA after device deployment shows near-complete cessation of flow. $C$, DSA at 6 months shows aneurysm recanalization. $D$, Photomicrograph $(\mathrm{HE}$, original magnification $\times 100)$ shows concave connective tissue across the aneurysm neck (block arrow).

\section{Histomorphometry and Analysis}

Two experienced observers evaluated the histologic sections, as described in a previous publication. ${ }^{4}$ The tissues within the aneurysmal dome were categorized as the following: 1) unorganized thrombus (fresh thrombus or poorly organized thrombus), 2) organized thrombus or organized tissues (connective tissue, which completely replaced the thrombus within the aneurysm dome), and 3) collagenized connective tissue (diffuse and attenuated collagenous matrix as well as less cellular and vascularized tissue within the connective tissue). Evaluation of aneurysm neck coverage was performed to see whether there was organized connective tissue across the neck continuously.

In this descriptive study, we did not perform any formal statistical analysis.

\section{Results}

\section{Angiographic Findings after Treatment}

Grade 1 (complete immediate cessation of intra-aneurysmal flow) was noted in 7 (29\%) of 24 cases. Thirteen (54\%) of 24 aneurysms showed near-complete cessation of flow (grade 2). Grades 3 (persistent flow with moderate-to-marked stasis), and 4 (persistent flow with no or minimal stasis) were noted in $2(8 \%)$ and $2(8 \%)$ cases, respectively.

Follow-up angiography was performed at 1 month $(n=6)$, 3 months $(n=6), 6$ months $(n=6)$, and 1 year $(n=6)$. Using the longest duration of implantation for final recanalization assessment, we noted complete occlusion in 8 (33\%) of 24 cases. Fourteen (58\%) cases showed near-complete aneurysm occlusion. Only 2 (8\%) cases had incomplete occlusion. Stable aneurysm occlusion was present in 7 (29\%) of 24 cases (Fig 2), $14(58 \%)$ cases showed progressive occlusion, and 3 (12\%) cases demonstrated recanalization (Fig 3).

\section{Histologic Findings}

Conventional histopathology showed that at 1 month, 3 of 6 aneurysm domes were completely filled with loose connective tissue; the remaining 3 aneurysms showed relatively small areas of unorganized thrombus in the dome. Five of 6 cases showed organized connective tissue across aneurysm neck. Mixed inflammatory infiltrate adjacent to the device along the aneurysm neck area was noted in 1 aneurysm.

At 3 months, 5 of 6 aneurysms demonstrated loose connective tissue completely occupying the aneurysm dome. There was organized connective tissue covering the aneurysm neck in all cases; each one also showed collagen deposition (Fig 2D). Localized chronic inflammatory infiltrate along the struts was noted in 2 aneurysms. Multinuclear giant cell infiltrate surrounding the device within the dome was observed in all 6 subjects.

Localized chronic inflammation within the aneurysm lumen was noted in 3 aneurysms at 6 months. Multinuclear giant cells surrounding the device within the aneurysm were shown in 1 subject. All 6 aneurysms had loose connective tissue occupying the aneurysm dome; there was organized connective tissue across the aneurysm neck. Part of the aneurysm 
cavity was filled with unorganized thrombus or was empty. In 1 case the aneurysm cavity was open to the parent artery (Fig 3D).

At 1 year, 6 of 6 aneurysms showed loose connective tissue within the aneurysm dome and organized connective tissue across the neck. Three incompletely occluded aneurysms also showed poorly organized or unorganized thrombus within the aneurysm cavity near the neck area. Local inflammation was noted in 2 aneurysms.

\section{Discussion}

In this study, we describe the in vivo performance of the WEB device, an intrasaccular aneurysm embolization device designed to provide a stable flow-disrupting high-attenuation metallic mesh across the aneurysm neck. We demonstrated that the device was readily placed into saccular aneurysms through standard microcatheters and filled the sac completely or nearly completely in a high proportion of cases. Complete or near-complete cessation of intra-aneurysmal flow was noted frequently within minutes after placement. The durability of the aneurysm occlusion was excellent and was similar to that noted with platinum coils in this same animal model. These data suggest that the WEB device might be used as a stand-alone device for treatment of a wide array of saccular aneurysms, irrespective of rupture status, because immediate cessation of flow was common and irrespective of parent artery geometry, because the device was placed in an intrasaccular location.

Multiple other flow-disrupting devices have been tested in preclinical models and have been applied in small clinical series. ${ }^{1-22}$ These other flow-disruption devices comprise tubular intraluminal metallic devices, which require dual antiplatelet therapy. Furthermore, while these devices have great utility in fusiform and wide-neck aneurysms that are not coilable, they are limited in cases of bifurcation anatomy and in vascular beds with eloquent perforating arteries. The WEB device overcomes these shortcomings yet still offers a high degree of neck coverage. Another potential advantage of this type of device compared with coils is that the actual flow inside the aneurysm can be visualized rather than relying on the surrogate radiographic presence of coils to assess flow stagnation. This could be a particularly important factor in ruptured cases. While not suitable for fusiform and wide-neck aneurysms (neck > dome), the WEB device offers a complementary approach that can potentially address most aneurysms.

In addition, the device was designed as a unitary structure so that it might provide a construct that would resist compaction, which is 1 of the primary drawbacks of embolic coils. Well-described in the literature, coil compaction is particularly a problem in high-flow (eg, terminal, bifurcation) aneurysms, resulting in high recanalization and retreatment rates. ${ }^{27-30}$ Finally, the company has an improved second generation of this device that is undergoing preclinical testing.

The exact mechanism underlying the long-term occlusion seen with intraluminal flow-disruption devices remains unknown, but it is usually a delayed phenomenon. Delayed occlusion may result from stasis and resultant thrombosis, from growth of endothelium across the metallic struts, or from both processes. The mechanism of occlusion with the WEB device, because it is nearly immediate in most cases, likely is that of flow disruption and resultant intrasaccular stasis and thrombosis. It is likely that the disruption of flow reduced inlet flow velocities below the thrombotic threshold velocity for the material, thus initiating thrombus formation. Computation flow studies would be useful to confirm this hypothesis. ${ }^{16}$

This study had several limitations. We did not include a concurrent control group of aneurysms treated by alternative therapies, such as the platinum MicroCoil system (Micrus). Our group has extensive experience in this model, showing rates of complete and near-complete aneurysm occlusion with coils on the order of $85 \%$ (unpublished data from our group). In the current study, the total rate of complete and near-complete aneurysm occlusion was similar to that of coils. The typical shape of the aneurysms in this model is relatively homogeneous, so the performance of the WEB device in other aneurysm shapes remains unknown. The maximum aneurysm dimension and neck width are relatively small compared with those in many human aneurysms, so the performance of the device in this model cannot be directly extrapolated to clinical use. The analysis of immediate flow disruption was based on a scale not previously validated.

\section{Conclusions}

The WEB device in elastase-induced aneurysms demonstrated promising rates of immediate and long-term aneurysm occlusion.

\section{References}

1. Kallmes DF, Williams AD, Cloft HJ, et al. Platinum coil-mediated implantation of growth factor-secreting endovascular tissue grafts: an in vivo study. Radiology 1998;207:519-23

2. Kallmes DF, Fujiwara NH, Yuen D, et al. A collagen-based coil for embolization of saccular aneurysms in a New Zealand white rabbit model. AJNR Am J Neuroradiol 2003;24:591-96

3. De Gast AN, Altes TA, Marx WF, et al. Transforming growth factor betacoated platinum coils for endovascular treatment of aneurysms: an animal study. Neurosurgery 2001;49:690-94

4. Dai D, Ding YH, Danielson MA, et al. Histopathologic and immunohistochemical comparison in human, rabbit, and swine aneurysms embolized with platinum coils. AJNR Am J Neuroradiol 2005;26:2560-68

5. Kallmes DF, Fujiwara NH. New expandable hydrogel-platinum coil hybrid device for aneurysm embolization. AJNR Am J Neuroradiol. 2002;23:1580-88

6. Ding Y, Dai D, Lewis D, et al. Angiographic and histologic analysis of experimental aneurysms embolized with platinum coils, Matrix, and HydroCoil. AJNR Am J Neuroradiol 2005;26:1757-63

7. Dai D, Ding YH, Danielson MA, et al. Endovascular treatment of experimental aneurysms by use of fibroblast-coated platinum coils: angiographic and histopathological study. Stroke 2007;38:170-76

8. Fujiwara NH, Kallmes DF. Healing response in elastase-induced rabbit aneurysms after embolization with a new platinum coil system. AJNR Am J Neuroradiol 2002;23:1137-44

9. Dai D, Ding YH, Danielson MA, et al. Endovascular treatment of experimental aneurysms with use of fibroblast transfected with replication-deficient adenovirus containing bone morphogenetic protein-13 gene. AJNR Am J Neuroradiol 2008;29:739-44

10. Killer M, Kallmes DF, McCoy MR, et al. Angiographic and histologic comparison of experimental aneurysms embolized with hydrogel filaments. AJNR Am J Neuroradiol 2009;30:1488-95. Epub 2009 May 27

11. Kallmes DF, Ding YH, Dai D, et al. A new endoluminal, flow-disrupting device for treatment of saccular aneurysms. Stroke 2007;38:2346-52

12. Kallmes DF, Ding YH, Dai D, et al. A second-generation, endoluminal, flowdisrupting device for treatment of saccular aneurysms. AJNR Am J Neuroradiol 2009;30:1153-58

13. Hans FJ, Krings T, Moller-Hartmann W, et al. Endovascular treatment of experimentally induced aneurysms in rabbits using stents: a feasibility study. Neuroradiology 2003;45:430-34

14. Sadasivan C, Cesar L, Seong J, et al. Treatment of rabbit elastase-induced aneurysm models by flow diverters: development of quantifiable indexes of device performance using digital subtraction angiography. IEEE Trans Med Imaging 2009;28:1117-25 
15. Augsburger L, Farhat M, Reymond P, et al. Effect of flow diverter porosity on intraaneurysmal blood flow. Klin Neuroradiol 2009;19:204-14

16. Radaelli AG, Augsburger L, Cebral JR, et al. Reproducibility of haemodynamical simulations in subject-specific stented aneurysm model: a report on the Virtual Intracranial Stenting Challenge 2007. J Biomech 2008 19;41:2069-81. Epub 2008 Jun 25

17. Wanke I, Forsting M. Stents for intracranial wide-necked aneurysms: more than mechanical protection. Neuroradiology 2008;50:991-98

18. Fiorella D, Albuquerque FC, Deshmukh VR, et al. Endovascular reconstruction with the Neuroform stent as monotherapy for the treatment of uncoilable intradural pseudoaneurysms. Neurosurgery 2006;59:291-300

19. Saatci I, Cekirge HS, Ozturk MH, et al. Treatment of internal carotid artery aneurysms with a covered stent: experience in 24 patients with mid-term follow-up results. AJNR Am J Neuroradiol 2004;25:1742-49

20. Lylyk P, Ferrario A, Pasbon B, et al. Buenos Aires experience with the Neuroform self-expanding stent for the treatment of intracranial aneurysms. $\mathrm{J} \mathrm{Neu}$ rosurg 2005;102:235-41

21. Fiorella D, Albuquerque FC, Deshmukh VR, et al. Usefulness of the Neuroform stent for the treatment of cerebral aneurysms: results at initial (3-6 mo) follow-up. Neurosurgery 2005;56:1191-201

22. Fiorella D, Lylyk P, Szikora I, et al. et al. Curative cerebrovascular reconstruction with the Pipeline embolization device: the emergence of definitive endovascular therapy for intracranial aneurysms. J Neurointervent Surg 2009;1:56-65
23. Turowski B, Macht S, Kulcsár Z, et al. Early fatal hemorrhage after endovascular cerebral aneurysm treatment with a flow diverter (SILK- Stent): do we need to rethink our concepts? Neuroradiology. 2011;53;37-41

24. Higashida RT, Hieshima GB, Halbach VV, et al. Intravascular detachable balloon embolization of intracranial aneurysms: indications and techniques. Acta Radiol Suppl 1986;369:594-96

25. Altes TA, Cloft HJ, Short JG, et al. 1999 ARRS Executive Council Award: creation of saccular aneurysms in the rabbit-a model suitable for testing endovascular devices. AJR Am J Roentgenol 2000;174:349-54

26. Fujiwara NH, Cloft HJ, Marx WF, et al. Serial angiography in an elastaseinduced aneurysm model in rabbits: evidence for progressive aneurysm enlargement after creation. AJNR Am J Neuroradiol 2001;22:698-703

27. Hayakawa M, Murayama Y, Duckwiler GR, et al. Natural history of the neck remnant of a cerebral aneurysm treated with the Guglielmi detachable coil system. J Neurosurg 2000;93:561-68

28. Murayama Y, Nien YL, Duckwiler GR, et al. Guglielmi detachable coil embolization of cerebral aneurysms: 11 years' experience. J Neurosurg 2003;98:959-66

29. Yoshino Y, Niimi Y, Song JK, et al. Endovascular treatment of intracranial aneurysms: comparative evaluation in a terminal bifurcation aneurysm model in dogs. J Neurosurg 2004;101:996-1003

30. Raymond J, Leblanc P, Desfaits AC, et al. In situ beta radiation to prevent recanalization after coil embolization of cerebral aneurysms. Stroke 2002;33: $421-27$ 